106 EFFECT OF A SINGLE SHORT ELECTROSTIMULATION SESSION OF THE FIBULARIS MUSCLES ON DYNAMIC POSTURAL STABILITY AND EVERTOR MUSCLES STRENGTH

${ }^{1}$ François Fourchet, ${ }^{2}$ Artiom Ganchine, ${ }^{1}$ Antoine Seurot, ${ }^{1}$ Nicolas Le Coroller, ${ }^{1}$ Guillaume Servant, ${ }^{1,3}$ Boris Gojanovic. 'Swiss Olympic Medical Center, Hôpital de La Tour, Meyrin, Geneva, Switzerland; ' Faculty of Medicine, Geneva University, Geneva, Switzerland; ${ }^{3}$ Interdisciplinary Center for Adolescent Sports Medicine, Woman-Mother-Child Department (DFME), CHUV, Lausanne, VD, Switzerland

10.1136/bjsports-2021-IOC.99

Background The fibularis muscles are key elements of the ankle evertor group (evertors). The weakness of evertors is one of the main cause of ankle sprains. Electrostimulation training can increase the maximum voluntary force of contraction by neural adaptation to a healthy skeletal muscle.

Objective To assess whether a single electrostimulation session of the fibularis muscles could impact dynamic postural stability and evertor strength.

Design Single-blind randomised controlled trial.

Setting Motion analysis laboratory in a hospital facility.

Patients (or Participants) Sixteen healthy male and female volunteers, randomly assigned to an experimental $(\mathrm{EXP}, \mathrm{n}=8)$ or control (Control, $\mathrm{n}=8$ ) group.

Interventions (or Assessment of Risk Factors) Participants in EXP received a single electrostimulation strengthening session for 3 minutes on the dominant side (DOM) fibularis muscles. Control participants received a sham electrostimulation on DOM with similar modalities.

Main Outcome Measurements Dynamic postural balance was assessed on DOM and non-dominant (NDOM) limbs using the modified Star Excursion Balance Test (SEBT). A composite score (CS in \%) was calculated. Evertor strength (in N) was assessed with three maximal isometric voluntary contractions on an isokinetic dynamometer.

Results EXP displayed a significant increase in SEBT-CS on DOM $(97.7 \pm 5.9 \%$ vs $96.1 \pm 7.4 \%, \mathrm{p}<0.05)$ and NDOM $(97.6$ $\pm 7.2 \%$ vs $95.6 \pm 7.4 \%, \mathrm{p}<0.01$ ), while these parameters did not change in Control. EXP also displayed a significant increase in Evertors isometric strength on DOM $(25.0 \pm 7.0 \mathrm{~N}$ vs $23.1 \pm 6.8 \mathrm{~N}, \mathrm{p}<0.05)$ and $\mathrm{NDOM}(26.8 \pm 6.0 \mathrm{~N}$ vs 22.9 $\pm 5.2 \mathrm{~N}, \mathrm{p}<0.001)$. In Control, evertor isometric strength increased on DOM $(24.9 \pm 7.4 \mathrm{~N}$ vs $23.1 \pm 6.8 \mathrm{~N}, \mathrm{p}<0.01)$, but remained unchanged on NDOM.

Conclusions A short single fibularis elecrostimulation session on one leg appeared to improve dynamic postural stability on both sides and evertors strength on the stimulated side only, suggesting a global post-activation potentiation effect of this intervention and a possible additional mirror effect on dynamic postural stability. These findings may be of interest for preventing ankle sprain.

\section{DOES ANKLE INSTABILITY ALTER MUSCLE ACTIVATION OF LOWER EXTREMITY AND GROUND REACTION FORCE DURING LANDING? A META-ANALYSIS}

1,25unghe $\mathrm{Ha},{ }^{1,2}$ Hyunggyu Jeon, ${ }^{1,2,3,4}$ Sae Yong Lee. 'Department of Physical Education, Yonsei University, Seoul, South Korea (Republic of); ${ }^{2}$ Frontier Research Institute of Convergence Sports Science, Yonsei University, Seoul, South Korea (Republic of); ${ }^{3}$ Yonsei Institute of Sports Science and Exercise Medicine, Yonsei University, Seoul, South Korea (Republic of); ${ }^{4}$ Institute of Convergence Science, Yonsei University, Seoul, South Korea (Republic of)

10.1136/bjsports-2021-IOC. 100
Background Altered muscle activation pattern and ground reaction force (GRF) in those with ankle instability (AI) should be clearly investigated in order to incorporate this information to retrain AI patient.

Objective To investigate muscle activation characteristics of AI patient and GRF pattern.

Data Sources Relevant studies were searched from PubMed, CINAHL, SPORTDiscus, and Web of Science through May 2019. Combination of keywords ankle instability, chronic ankle instability, ankle sprain, biomechanics, kinetics, electromyography, and landing were used to search relevant studies.

Study Selection Inclusion criteria for study selection were: 1) subjects with chronic ankle instability, functional instability, mechanical instability or recurrent ankle sprains; 2) the primary outcomes consisted of muscle activation of the lower extremity and GRF during landing; 3) peer-reviewed articles with full-text; and 4) providing appropriate information, which is mean, standard deviation, and sample size to re-analyze data.

Data Extraction Extracted data included muscle activation of the lower extremity (root mean square; integral EMG; mean), the magnitude, and time to peak GRF and was used to calculate standardized mean differences (SMD) with 95\% confidence intervals (CIs).

Data Synthesis A total of twelve relevant studies (Oxford Centre for Evidence-Based Medicine level 3b) included in this study. The peroneal muscle was less activated in AI compared to control before landing $(\mathrm{SMD}=-0.59, \mathrm{p}<0.01$, CIs $=-0.91$, $0.27)$. AI had greater peak vertical GRF $(S M D=0.21, p=0.03$, CIs $=0.02,0.41)$ and exhibited shorter time to peak vertical GRF (SMD $=-0.63, \mathrm{p}<0.01, \mathrm{CI}=-0.85,-0.41)$ than those of control during landing $(\mathrm{SMD}=-0.63, \mathrm{p}<0.01, \mathrm{CI}=-0.85$, $0.41)$.

Conclusions Muscle recruitment training of the peroneal muscle may diminish the risk of the recurrent ankle sprain in addition to other lower limb injuries. The peroneal muscle could provide a sufficient range of plantar flexion to decrease vertical GRF and eversion of the subtalar joint. Therefore, peroneal muscle training may be a key factor to retrain for the altered landing strategy resulting in ankle instability.

\section{ASSOCIATION BETWEEN ANKLE SPRAIN HISTORY AND ANKLE SPRAIN INCIDENCE IN NATIONAL BASKETBALL ASSOCIATION GAMES}

1,2,3 Mackenzie Herzog, ${ }^{1,2}$ Christina Mack, ${ }^{1,2}$ Nancy Dreyer, ${ }^{3}$ Erik Wikstrom, ${ }^{3}$ Darin Padua 4,5,6Mininder Kocher, ${ }^{2,3}$ Steve Marshall. 'IQVIA Injury Surveillance and Analytics, Durham, USA; ${ }^{2}$ University of North Carolina at Chapel Hill Department of Epidemiology, Chapel Hill, USA; ${ }^{3}$ University of North Carolina at Chapel Hill Department of Exercise and Sport Science, Chapel Hill, USA; ${ }^{4}$ The Micheli Center for Sports Injury Prevention, Boston, USA; ${ }^{5}$ Boston Children's Hospital Department of Orthopedics, Boston, USA; ${ }^{6}$ Harvard Medical School Department of Orthopaedic Surgery, Boston, USA

\subsection{6/bjsports-2021-IOC.101}

Background Ankle sprains have one of the highest recurrence rates among all lower extremity injuries in sports.

Objective Assess the association between ankle sprain history in the past year and incident ankle sprain in games.

Design Retrospective Cohort Study

Setting U.S. National Basketball Association (NBA)

Participants All players who participated in an NBA game over 2 or more seasons from 2013-14 through 2016-17. 
Assessment of Risk Factors History of ankle sprain in the past year was defined in a time-dynamic manner as any bilateral ankle sprain (game or non-game) in the 365 days prior to the game of interest. Hazard ratios (HR) and $95 \%$ confidence intervals (CI) were calculated while controlling for years in the NBA, body mass index, and average NBA minutes played in the prior year.

Main Outcome Measurements All acute-onset ankle sprains reported in games were identified from the NBA standardized electronic medical record ( $\mathrm{n}=30$ teams).

Results Across this 4-season study, 482 incident ankle sprains were reported in NBA games among 681 players and $2,517,549$ player-minutes. Of the players that sustained an incident sprain, 44\% $(n=211)$ had at least one ankle sprain in the prior year. Compared to players with no sprains in the past year, the risk of incident ankle sprain increased with increasing number of prior ankle sprains; a $28 \%$ increase in risk $(\operatorname{adjHR}=1.28,95 \%$ CI $1.03,1.58)$ with one prior sprain, a $51 \%$ increase in risk $(\operatorname{adjHR}=1.51,95 \%$ CI 1.10, 2.04) with two prior sprains, and a $100 \%$ increase in risk (adjHR $=2.00,95 \%$ CI $1.31,2.94)$ with three or more prior sprains.

Conclusions History of ankle sprain in the past year was associated with increased risk of incident ankle sprain among NBA players. $44 \%$ of players had at least one ankle sprain (game or non-game) within one year prior to the incident sprain.

\section{AN UNSUPERVISED E-HEALTH SUPPORTED NEUROMUSCULAR TRAINING PROGRAM IS NOT EFFECTIVE IN THE PREVENTION OF RECURRENT ANKLE SPRAINS IN PATIENTS IN PRIMARY CARE: THE TRAPP- STUDY}

${ }^{1}$ Adinda Mailuhu, ${ }^{2}$ Evert Verhagen, ${ }^{1} J o h n$ Van Ochten, ${ }^{1}$ Patrick Bindels, 'Sita BiermaZeinstra, ${ }^{1}$ Marienke Van Middelkoop. 'Department of General Practice, Erasmus MC, University Medical Centre, Rotterdam, Netherlands; ${ }^{2}$ Department of Health Sciences and EMGO Institute for Health and Care Research, VU University Medical Center, Amsterdam, Netherlands

\subsection{6/bjsports-2021-IOC.102}

Background Ankle sprains are one of the most frequent injuries of the musculoskeletal system. No optimal treatment strategy has proven to be effective in general practice, however promising results were achieved in a preventive trial among athletes

Objective To examine the effectiveness of an unsupervised ehealth supported neuromuscular training program in combination with usual care in general practice compared to usual care alone in patients with acute lateral ankle sprains in general practice

Design Randomised Controlled Trial

Setting Primary care

Patients (or Participants) Patients (14-65 years) who visited a general practitioner with an acute lateral ankle sprain within three weeks of injury

Interventions (or Assessment of Risk Factors) The intervention group received, in addition to usual care, an unsupervised ehealth supported neuromuscular training program, and the control group received usual care alone

Main Outcome Measurements The primary outcome was selfreported recurrent sprains during follow-up. Secondary outcomes were ankle function (Ankle Function Score 0-100), pain in rest and during activity (numerical rating scale $0-10$ ), subjective recovery and return to the same type and level of sport.

Results 165 participants were included with a mean age of 38.3 (SD 14.2) years and $41.8 \%$ were male. No difference in the occurrence of a recurrent sprain was found between the intervention $(n=17 ; 20.7 \%)$ and control group $(n=20$; 24.1\%) (HR 1.14; 95\% CI 0.59-2.21). Also, no differences in secondary outcomes were found between groups. The compliance rate with the program was low (6.1\%).

Conclusions Although the recurrence rate of ankle sprains was relatively high, an unsupervised e-health supported neuromuscular training program is not effective in preventing recurrent sprains in patients with an acute lateral ankle sprain in general practice. More research is necessary to indicate the best treatment modality for this group of patients.

\section{\begin{tabular}{|l|l}
110 & UNDERSTANDING THE IMPACT OF ANKLE INJURIES
\end{tabular} AMONG CONTEMPORARY PRE-PROFESSIONAL DANCERS: INCIDENCE AND RISK FACTORS}

${ }^{1}$ Adinda Mailuhu, ${ }^{2}$ Rogier van Rijn, 1,2,3,4 Janine Stubbe, ${ }^{1}$ Sita MA Bierma-Zeinstra, ${ }^{1}$ Marienke van Middelkoop. 'Department of General Practice, Erasmus MC University Medical Center, Rotterdam, Netherlands; ${ }^{2}$ Codarts, University of the Arts, Rotterdam, Netherlands; ${ }^{3}$ PErforming artist and Athlete Research Lab (PEARL), Rotterdam, Netherlands: ${ }^{4}$ Rotterdam Arts and Sciences Lab (RASL), Rotterdam, Netherlands

\subsection{6/bjsports-2021-IOC.103}

Background Ankle injuries are common injuries among preprofessional dancers. An overview of the incidence and risk factors is however lacking.

Objective To examine the incidence and risk factors of ankle injuries among contemporary pre-professional dancers.

Design Prospective cohort study.

Setting Codarts University of the Arts, Rotterdam, The Netherlands.

Patients (or Participants) 91 first-year contemporary pre-professional dancers, from Bachelor Dance $(n=59)$ and Bachelor Dance Teacher $(n=32)$ were included.

Interventions (or Assessment of Risk Factors) At the start of the academic year all dancers completed a questionnaire, underwent a physical screening measuring the ankle range of motion (ROM) and dorsiflexion. During the academic year, all dancers completed monthly questionnaires on the occurrence of an ankle injury that were referred to in the Oslo Sports Trauma Research Center (OSTRC) questionnaire. Injuries were defined into substantial injuries (score $\geq 13$ on question 2 or 3 of OSTRC questionnaire), injuries leading to medical attention and to dance time-loss. Potential risk factors for an ankle injury included dancer characteristics, history of ankle injury in previous year, ankle ROM and dorsiflexion.

Main Outcome Measurements The number of self-reported ankle injuries during one academic year.

Results 17 (18.7\%) dancers reported an ankle injury, with a total of 33 injuries. Of these dancers reporting an ankle injury, $82.4 \%$ led to dance time-loss (mean number of 19.9 days unable to fully participate), $47.1 \%$ to medical attention and $41.2 \%$ were substantial injuries. No significant risk factors for ankle injuries could be identified. 